\title{
Melkersson-Rosenthal syndrome - a therapeutically challenging disease
}

\author{
Aleksandra Wnuk-Kłosińska ${ }^{1}$, Dorota Jenerowicz ${ }^{1}$, Monika Bowszyc-Dmochowska $^{1}$, Honorata Pietrzak-Kaczmarek ${ }^{2}$, \\ Zygmunt Adamski ${ }^{1}$
}

'Department of Dermatology, Poznan University of Medical Sciences, Poznan, Poland

${ }^{2}$ Department of Rheumatology and Internal Diseases, Poznan University of Medical Sciences, Poznan, Poland

Adv Dermatol Allergol 2021; XXXVIII (3): 533-536

DOI: https://doi.org/10.5114/ada.2020.92569

Melkersson-Rosenthal syndrome (MRS) is a rare disease characterised by recurrent orofacial swelling, recurrent facial paralysis, and a fissured tongue. However, cases in which the patient experiences all the three symptoms are relatively rare (in app. 8-25\% of all cases). The clinical picture is dominated by incomplete and monosymptomatic forms [1-7]. The latest reports on MRS state that the disease annually affects from 0.2 to 80 per 100,000 people. However, the number of MRS incidences is believed to be higher becuase the syndrome is often misdiagnosed or diagnosed incompletely [6].

Typically, the MRS symptoms appear in the second and third decades of life, but they may also manifest regardless of age, also the paediatric population $[4,6,8]$. Some literature data suggest that there is no difference in the prevalence of the disease in both sexes [1], others indicate that MRS is more frequent in women (suspected impact of female sex hormones as an aetiopathogenetic factor) [6].

The most common symptom of MRS is swelling of the soft facial tissues (mainly the upper and lower lip), which is found in $75-100 \%$ of patients. The swelling is painless, appears suddenly, and lasts for a few days. However, with time it tends to recur and becomes chronic $[1,4]$. Oedema may also include mucous membranes of the cheeks and palate, gums, and the eyelids, forehead, and chin areas [4, 6]. Facial nerve palsy is found in 30-90\% of patients. It is usually one-sided, recurrent, and can be partial or complete $[1,4]$. It is associated with a worse prognosis as compared to Bell's paralysis [9]. The third symptom, i.e. a fissured tongue, is observed in $30-77 \%$ of patients. Its presence in the healthy population is estimated to range from $5 \%$ to $10 \%[1,4]$. In MRS, the tongue is enlarged, swollen, and stinging, with a marked middle furrow and cracks on the dorsal surface. The symptom is sometimes accompanied by taste disorders, impaired salivary secretion, and speech difficulties. In addition to the classic triad of MRS symptoms, swollen lymph nodes, mainly in the chin and submandibular areas, can be observed [4]. Oedema of the genital and anal regions is rare but has also been reported in a few MRS patients [7, 10].

The diagnosis of MRS is made by physical findings and history. A biopsy may be necessary to confirm the diagnosis of this rare disease [2].

The aim of this case report was to present the patient suffering from ankylosing spondylitis in whom first MRS symptoms appeared during treatment with etanercept. With the purpose to establish the diagnosis and prepare the case description the authors used diagnostic methods: biopsy, patch tests, laboratory tests, and diagnostic imaging.

A 55-year-old woman presented with a persistent oedema of the upper lip, which had occurred 5 months earlier and showed no response to treatment with antihistamines, glucocorticosteroids, and topical tacrolimus. The patient had been suffering from ankylosing spondylitis for over 20 years, accompanied by arterial hypertension and hypothyroidism. Individual and family history of dermatological diseases were negative. The patient remained under constant rheumatological care. In the course of the underlying disease, she was treated with prednisone, non-steroidal anti-inflammatory drugs, sulfasalazine, adalimumab, and etanercept (the latter being administered since 2012 - the last treatment round had been conducted for the 4 months before her visit to our Department).

On admission, the patient's enlarged swollen upper lip and fissured tongue (Figures 1 A, B) could immediately be noticed in the physical examination. There were no changes of oral mucosa, no enlargement of peripheral lymph nodes, and no features of facial nerve palsy. The patient reported periodic burning of the lips and tongue area. Laboratory examinations of blood and urine re-

Address for correspondence: Dorota Jenerowicz, Department of Dermatology, Poznan University of Medical Sciences, 49 Przybyszewskiego St, Poznan, Poland, e-mail: djenerowicz@yahoo.com Received: 25.09.2019, accepted: 17.10.2019. 

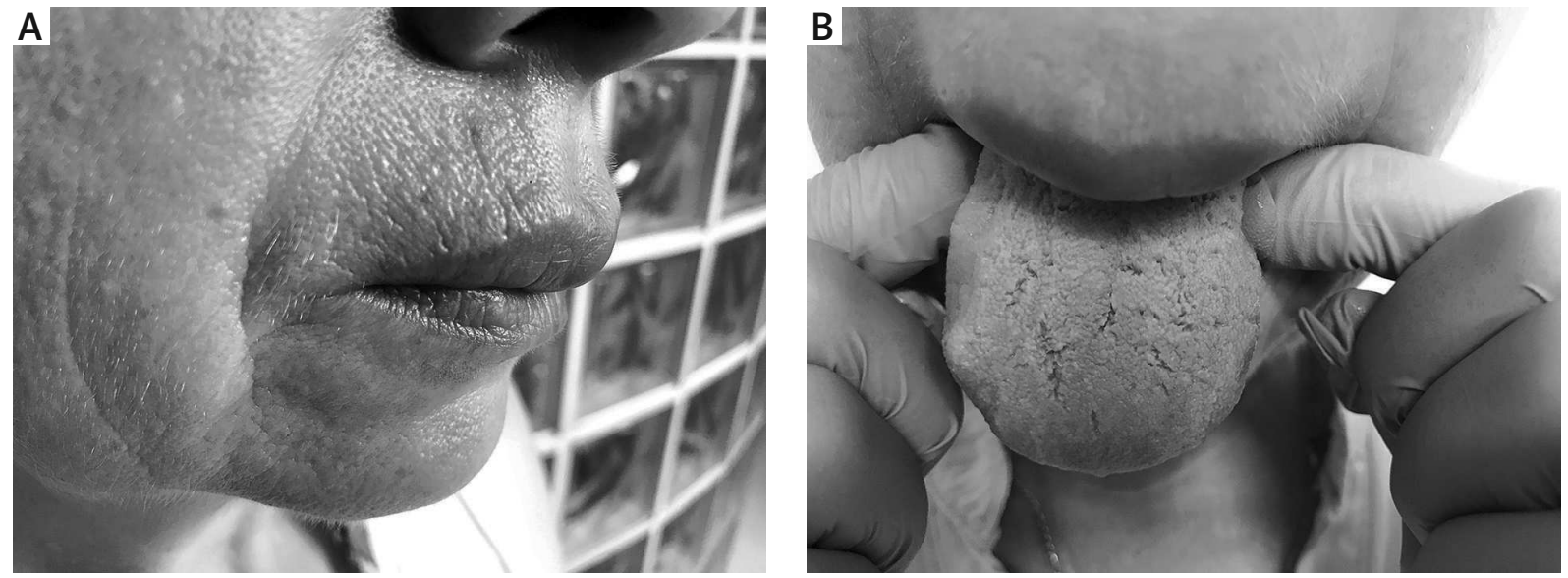

Figure 1 A, B. The patient's enlarged swollen upper lip and fissured tongue before treatment
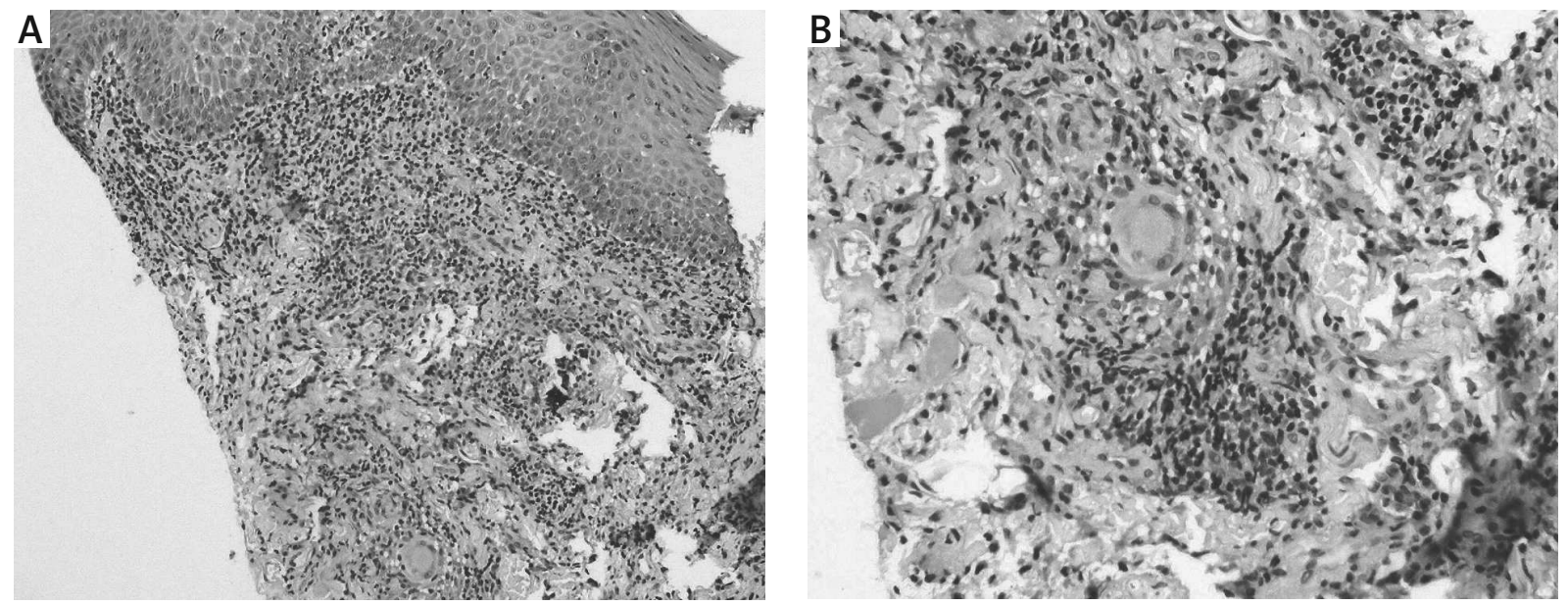

Figure 2. A - Thickened, oedematous epithelium and dense chronic inflammatory infiltrate in the lamina propria (100x). B - Small granuloma with single giant cell in the deeper part of the inflammatory infiltrate $(200 x)$

vealed no abnormalities. Patch tests performed with a European Standard Series of potential contact allergens were negative. Also, abdomen ultrasound and thyroid ultrasound, as well as chest X-ray were normal.

An excision from the upper lip, more precisely from the joint line of the vermillion border and the mucosa, was taken. Histopathological examination revealed thickened, oedematous epithelium with a dense inflammatory lymphocytic-plasmacytic-histiocytic infiltrate in the lamina propria. In its deeper layers, formation of small granulomas with a single giant cell could be observed (Figures 2 A, B). On the basis of the clinical picture as well as the histopathological examination, the MRS was diagnosed.

A decision to modify the biological treatment was made. It was driven by the suspicion of induction of the MRS by etanercept, and the literature reports on beneficial results of infliximab and adalimumab in the MRS treatment $[3,5,11]$ and their positive influence on the clinical activity of the underlying disease. Infliximab at a dose of 400 mg every 8 weeks was included to the treatment, which resulted in a significant improvement in the patient's joint ailments. However, because no significant improvement in the patient's dermatological condition could be observed after a 10-month therapy with infliximab, the patient was admitted to the Dermatology Clinic, where she was further diagnosed and qualified for the inclusion of additional treatment. At the hospital, the patient was also consulted with an ophthalmologist, who confirmed there were no contraindications to add a daily $250 \mathrm{mg}$ dose of chloroquine to the patient's treatment. Chloroquine treatment was continued on an outpatient basis, together with infliximab therapy. After 3 months, the patient achieved a gradual, partial improvement of her clinical condition, particularly in terms of subjective ailments such as burning (Figure 3 ).

The patient is now under constant dermatological and rheumatological care and continues to receive therapy with infliximab and chloroquine.

MR syndrome was first described in 1928 by Melkersson, who documented a case of a patient with co- 
occurring swelling of soft facial tissues and facial nerve palsy. Three years later, Rosenthal reported on the third symptom of the MRS triad, i.e. a fissured tongue [4, 5, 9]. Having the same histopathological images as the MRS, cheilitis granulomatosa, described in 1945 by Miescher, is often considered to be a monosymptomatic form of the MR syndrome [1, 2, 4, 6]. Other similarities between the two diseases are, among others, chronic and recurrent nature or resistance to treatment [1].

In 1985, Wiesenfeld et al. introduced the term orofacial granulomatosis (OFG), including cheilitis granulomatosa and MR syndrome to this group [12]. The term covers the spectrum of orofacial diseases that are characterised by the occurrence of soft tissue oedema around the face and mouth, and whose histopathological examination confirms the presence of non-necrotising granulomas [13]. A similar clinical picture may be a manifestation of diseases such as sarcoidosis or Crohn's disease [6, 13]. According to some authors, cheilitis granulomatosa is sometimes present several years before the intestinal changes in Crohn's disease can be seen, and in $0.5 \%$ of all cases it is a parenteral manifestation of this disease [9].

The exact aetiology of MRS is not known yet. However, its origins are believed to be influenced by genetic, infectious, and allergic factors [6, 8, 9]. Some literature research reports that autoimmune dysregulation may play a part in the aetiopathogenesis of MR syndrome $[1,8,9]$. Cases of patients suffering from both MRS and autoimmune diseases, such as multiple sclerosis or Hashimoto's thyroiditis, are also described $[8,11]$. In addition, the presence of granulomas in the histopathological image makes it possible to consider MRS as a disease from the group of sarcoidosis or Crohn's disease [7, 11].

A possible relationship between etanercept treatment for ankylosing spondylitis and MR syndrome is assumed in the patient presented in the study. In 2013, Gaudio et al. described an interesting case of a 42-year-old woman with MRS, who was treated with etanercept for psoriatic arthritis [11]. This was probably the first and the only report of this kind in the medical literature. Etanercept, like infliximab and adalimumab, belongs to the tumour necrosis factor $\alpha$ (TNF- $\alpha)$ inhibitors. TNF- $\alpha$ is a pro-inflammatory cytokine produced, among others, by monocytes, macrophages, and T lymphocytes. With its pleiotropic effect, it plays a significant role in the formation of granulomas $[3,11]$. It seems that in anti-TNF-treated patients, sarcoid granulomatous reactions of various kinds are observed more frequently for a soluble receptor in the dimeric form than for the anti-TNF- $\alpha$ monoclonal antibody. This may be caused by etanercept's different biological properties in comparison to medicines such as infliximab or adalimumab [11].

Due to its unclear aetiology, treatment of the MR syndrome is difficult and sometimes associated with resistance to the applied pharmacotherapy and risk of recurrence of symptoms [2]. So far, no standardised therapy

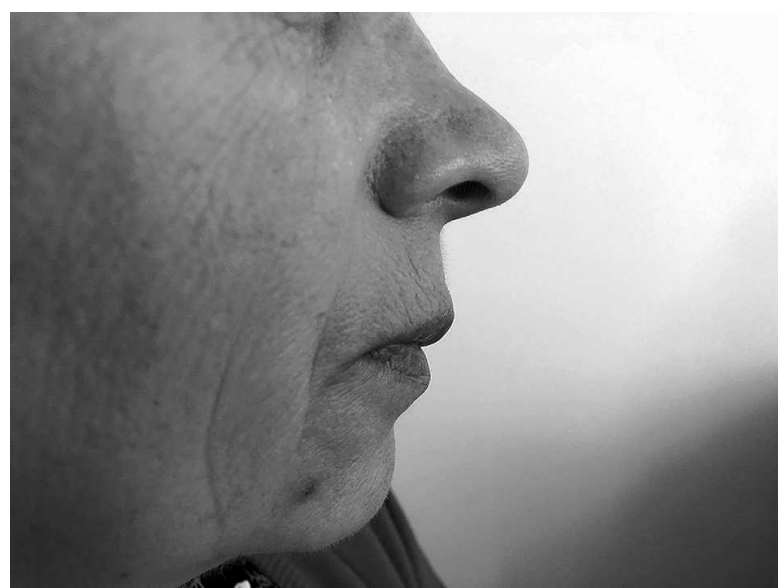

Figure 3. Patient's clinical condition after 3 months of chloroquine therapy

scheme has been developed, and the choice of treatment is largely based on reports from already published cases [9]. The treatment uses, among others, non-steroidal anti-inflammatory drugs, antihistamines, or glucocorticoids, applied both locally and systemically [2, 8]. However, such therapy did not produce any improvement in the clinical condition of the patient described in the study. Immunosuppressive drugs, such as methotrexate, antibiotics, or antimalarials, are also used in the treatment $[7,8]$. One of the latest reports describes the case of an 8-year-old child with the classic triad of MR symptoms, whose clinical condition significantly improved after intravenous treatment with immunoglobulins [8]. An alternative therapy might be surgical intervention, but this is only made in cases of recurrent changes and resistance to conservative treatment or in situations of a significant cosmetic defect of the face area [2, 5].

It is noteworthy that there are literature reports on successful attempts at biological treatment of MR syndrome, namely use of infliximab and adalimumab. This applies to patients both with a fully symptomatic form of the disease and with lesions limited to cheilitis granulomatosa [3, 5, 9]. The first successful trial of treatment of cheilitis granulomatosa with infliximab was by Barry et al. Infliximab therapy at a dose of $3 \mathrm{mg} / \mathrm{kg}$ was added to the treatment of a 24-year-old woman with a 5-year history of persistent oedematous lips, after prior failure of pharmacotherapy, including minocycline, erythromycin, clofazimine, and topically applied tacrolimus and triamcinolone injections. After the second infusion, the patient's clinical condition improved considerably. The infliximab therapy was continued with an increased dosage of $5 \mathrm{mg} / \mathrm{kg}$ infusions every 8 weeks. No sign of relapse was observed in the patient [14]. De Moll et al. described the case of a 69-year-old woman with a full triad of MR symptoms, who was treated with adalimumab. The first signs of improvement in the patient's clinical condition 
could be seen after 3 months of therapy at a dose of $40 \mathrm{mg}$ per week. The patient was closely observed for 11 months, and no recurrence of the symptoms was reported during this time [3].

However, for the patient described in the study, the 10-month infliximab therapy implemented due to coexisting ankylosing spondylitis did not bring satisfactory improvement of her dermatological condition. It was then decided to add chloroquine to the treatment. Chloroquine belongs to the group of antimalarials that show immunomodulatory, anti-inflammatory, antiproliferative, and photoprotective effects. With the accumulation of antimalarials in lysosomes, the $\mathrm{pH}$ increases. This inhibits the formation of antigenic peptides with class II molecules of the major histocompatibility complex, requisite to stimulate CD4+ T. As a result, the immune response against autoantigenic peptides is suppressed. Thanks to their properties, antimalarials, including chloroquine, are widely used in the therapy of various skin diseases [15, 16]. They are also recommended as a therapy of orofacial granulomatosa and the MRS [1, 4, 7]. Lalosevic et al. reported a case of orofacial granulomatosa in a 12-yearold girl treated with intravenous pulse corticosteroids and chloroquine. The patient presented a good clinical response to this therapy [15]. Inclusion of chloroquine in the treatment of the patient described in our study resulted in the patient's gradual improvement after 3 months of the therapy.

The recurrent and persistent nature of the MRS symptoms is often associated with considerable discomfort and reduced quality of patients' lives. Despite use od the latest treatments, MRS remains a challenge to clinicians, mainly because of its unknown aetiology and rare occurrence.

\section{Conflict of interest}

The authors declare no conflict of interest.

\section{References}

1. Antoszczyk G, Obtułowicz A, Czarnobilska E, Wojas-Pelc A. Melkersson-Rosenthal syndrome - diagnostic and therapeutic problems. Prz Lek 2008; 65: 390-2.

2. Cancian M, Giovannini S, Angelini A, et al. MelkerssonRosenthal syndrome: a case report of a rare disease with overlapping features. Allergy Asthma Clin Immunol 2019; 15: 1. https://doi.org/10.1186/s13223-018-0316-z.

3. de Moll EH, Lebwohl MG. Melkersson-Rosenthal syndrome successfully treated with adalimumab. Cutis 2018; 101: 122-4.

4. Jakubowicz O, Żaba R, Czarnecka-Operacz M, Silny W. Difficulties connected with diagnosis of Melkersson-Rosenthal syndrome - case report. Adv Dermatol Allergol 2009; 26: 165-70.

5. Kakimoto C, Sparks C, White AA. Melkersson-Rosenthal syndrome: a form of pseudoangioedema. Ann Allergy Asthma Immunol 2007; 99: 185-9.

6. Savasta S, Rossi A, Foiadelli T, et al. Melkersson-Rosenthal syndrome in childhood: report of three paediatric cases and a review of the literature. Int J Environ Res Public Health 2019; 16: 1289.

7. Wickramasinghe N, Gunasekara CN, Fernando WS, et al. Vulvitis granulomatosa, Melkersson-Rosenthal syndrome, and Crohn's disease: dramatic response to infliximab therapy. Int J Dermatol 2012; 51: 966-8.

8. Fantacci C, Mariotti P, Miceli Sopo S, et al. Intravenous immunoglobulins in Melkersson-Rosenthal syndrome: a clinical and neuroimaging study. Pediatr Allergy Immunol 2018; 29: 881-3.

9. Wehl G, Rauchenzauner M. A systematic review of the literature of the three related disease entities cheilitis granulomatosa, orofacial granulomatosis and Melkersson-Rosenthal syndrome. Curr Pediatr Rev 2018; 14: 196-203.

10. Chu Z, Liu Y, Zhang H, et al. Melkersson-Rosenthal syndrome with genitalia involved in a 12-year-old boy. Ann Dermatol 2016; 28: 232-6.

11. Gaudio A, Corrado A, Santoro N, et al. Melkerssn-Rosenthal syndrome in a patient with psoriatic arthritis receiving etanercept. Int J Immunopathol Pharmacol 2013; 26: 229-33.

12. Wiesenfeld D, Ferguson MM, Mitchell DN, et al. Orofacial granulomatosis - a clinical and pathological analysis. Q J Med 1985; 54: 101-13.

13. Vassallo C, Rivetti N, Merlino M, et al. Effectiveness of surgical treatment of severe macrocheilia in a patient with orofacial granulomatosis. Clin Exp Dermatol 2017; 42: 887-9.

14. Barry O, Barry J, Langan S, et al. Treatment of granulomatous cheilitis with infliximab. Arch Dermatol 2005; 141: 1080-2.

15. Lalosevic J, Gajic-Veljic M, Nikolic M. Orofacial granulomatosis in a 12-year-old girl successfully treated with intravenous pulse corticosteroid therapy and chloroquine. Pediatr Dermatol 2017; 34: e324-7.

16. Rodriguez-Caruncho C, Bielsa Marsol I. Antimalarials in dermatology: mechanism of action, indications, and side effects. Actas Dermosifiliogr 2014; 105: 243-52. 